Revista de la Facultad de Ingeniería Industrial

Vol. 12(1): pp 27-31 (2009) UNMSM

ISSN: 1560-9146 (Impreso) / ISSN: 1810-9993 (Electrónico)

\title{
Tecnología de clasificación fina de mineral metálico y no metálico
}

Silvana Flores Chávez ${ }^{1}$ Alfonso Romero Baylón ${ }^{2}$ David Martínez Aguilar ${ }^{3}$

\section{RESUMEN}

El artículo describe una visión general sobre el uso del Cyclosizer, utilizado principalmente para la realización de pruebas experimentales de minerales metálicos y no metálicos en el procesamiento de minerales tales como: mineral aurífero y mineral de dolomita, respectivamente. El cyclosizer es un elutriador que separa una muestra en fracciones de tamaño específico por una técnica que depende de las fuerzas producidas por las velocidades relativas de las partículas y del fluido de elutriación. Difiere de una elutriación convencional en la que la acción de elutriación toma lugar en un ciclón hidráulico donde el fluido está girando y las fuerzas centrífugas muchas veces, debido a la gravedad, están actuando sobre las partículas.

Los modelos de fluidos dentro del ciclón son estables y los cambios en las condiciones del ambiente no son muy críticas como el procedimiento convencional para elutriación. También las altas fuerzas cortantes que son desarrolladas en el ciclón sobrepasan cualquier tendencia natural para que flocule el material fino y una excelente disposición de las partículas sea asegurada.

Palabra clave: Cyclosizer, Elutriador, tamaño.

TeChNOLOGY BY Fine PARTICLES CLASSIFICATION OF NO METALIC MINERALS

\section{ABSTRACT}

This article describes a general vision about the use of the Cyclosizer used mainly for the realization o experimental tests of metallic and non metallic minerals in the processing of minerals such as: gold bearing minerals and dolomite minerals, respectively. The cyclosizer is an elutriator that separates a specimen in specifically sized fractions using a technique that depends on the forces produced by the relative speed of the particles and the elutriation fluid. It is different from a conventional elutriation where the elutriation action takes place in a hydraulic cyclone where the fluid is spinning and the centrifuge forces are acting on the particles due to the gravity.

The fluid models in the cyclone are stable and the changes in the condition of the atmosphere are not as critical as in the case of the conventional processing for elutriation. Also, the high cutting forces that are developed in the cyclone exceed any natural tendency in order to floccule the fine material and assure an excellent disposition of the particles.

KEYwoRDs: Cyclosizer, Elitriador. Size.

\section{INTRODUCCIÓN}

El cyclosizer clasifica en fracciones simples todas las partículas que tienen una velocidad de sedimentación de un rango específico. El diámetro equivalente de "Stokes", de entre todas las partículas, van estar dentro de un rango debido a que todas tienen la misma densidad. Si la densidad varía entre las partículas, entonces, para mantener la validez de la Ley de "Stokes", cada fracción tendrá un rango diferente de tamaño para cada densidad de partículas.

En el presente trabajo el concepto de diámetro equivalente de "Stokes" es usado exclusivamente para describir el tamaño de partículas. Para las partículas dentro del rango del subtamizado, esta velocidad es descrita por la Ecuación de "Stokes" (1), que establece la velocidad de caída libre y el diámetro de partículas esféricas, cayendo bajo la influencia de la gravedad:

$$
V=\frac{d^{2} g\left(\rho_{s}-\rho_{f}\right)}{18 \mu}
$$

Donde:

$V=$ Velocidad de caída libre $(\mathrm{cm} / \mathrm{seg}$.

$\mathrm{d}=$ diámetro de partícula $(\mathrm{cm})$

$\mathrm{g}=$ aceleración debido a la gravedad $\left(\mathrm{cm} / \mathrm{seg}^{2}\right)$

$\rho_{\mathrm{s}}=$ Densidad de la partícula $(\mathrm{g} / \mathrm{cc})$

$\rho_{\mathrm{f}}=$ Densidad del fluido $(\mathrm{g} / \mathrm{cc})$

$\mu=$ Viscosidad del fluido (poise)

\section{FIGURA 1: CYCLOSIZER}

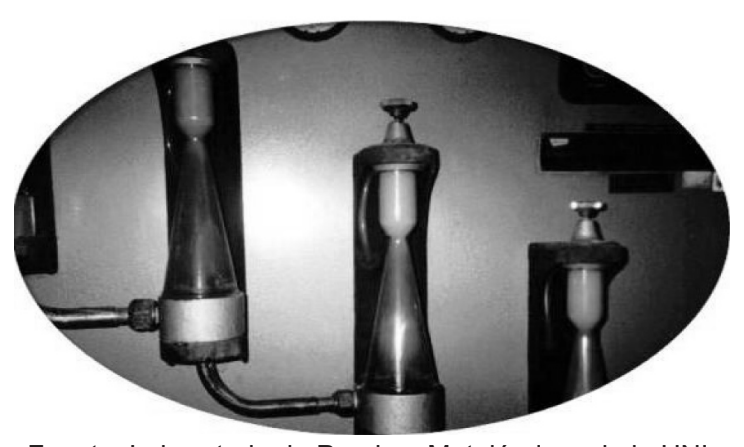

Fuente: Laboratorio de Pruebas Metalúrgicas de la UNI.

Ing. Metalurgista - Universidad Nacional Mayor de San Marcos;

Docente Investigador del IIGEO de la Universidad Nacional Mayor de San Marcos. Docente UNI. 
El cyclosizer M-6, fabricado por el Warman International Ltd., es un aparato de laboratorio de bastante precisión cuya función principal es reunir las partículas en rangos exactos de tamaños definidos dentro de los cuerpos de las cámaras del apex de los cinco ciclones que forman la base del cyclosizer. Estos están instalados en serie pero en posición invertida, poseen un diseño bastante original, ya que tiene un termómetro para medir la temperatura del tanque del agua, un manómetro para medir la presión con que bombea el agua, un rotámetro con graduaciones en milímetro para medir el flujo de agua. Para objeto de cálculos se deberá convertir en litros/minuto. Este cyclosizer opera con precisión en el intervalo de 9 a 14 litros/ minuto y posee un container (recipiente de muestras), caja de control del interruptor de la bomba y el timer para controlar el tiempo.

\section{MARCO CONCEPTUAL}

En el cyclosizer, la fuerza que produce la separación es la centrífuga. El típico ciclón hidráulico ilustrado consiste en una sección cilíndrica corta de cámara de vortex cerrada en un extremo y montado con una tubería de rebose axialmente (la salida del vortex) introduciéndose el cuerpo del ciclón. Hay un alimentador tangencial abierto. Ver Figura 2.

\section{FIGURA 2: CICLÓN}

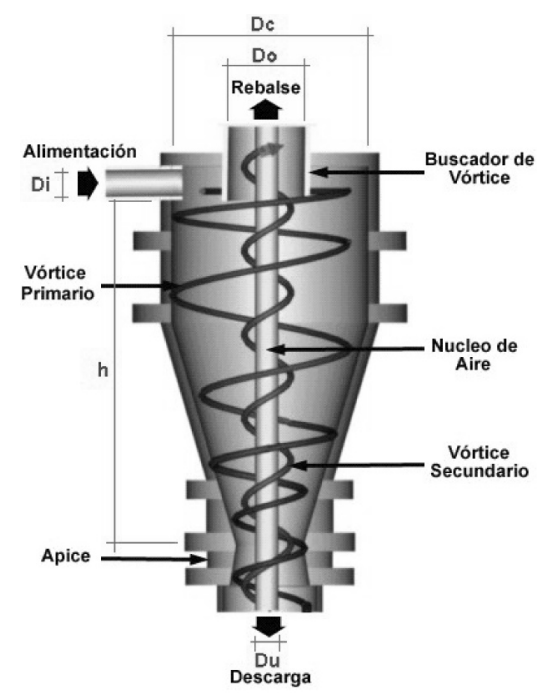

Fuente: Sepor INC “Ciclosizer For Sub-Sieve Sizing”.

De las muchas ecuaciones empíricas desarrolladas, (Lynch Yoshioka y Hotta, Dahistron, Tarjan Staos), la más reciente y representativa de todos los trabajos anteriores es la de L. R. Plitt. Ecuaciones 2 y 3.

\subsection{Modelo de Plitt}

$$
\begin{gathered}
d_{50}=\frac{35 D c^{0.46} D i^{0.6} D i^{1.21} \exp (0.063 \Phi)}{D u^{0.71} h^{0.38} Q^{0.45}\left(\rho_{s}-\rho_{l}\right)^{0.5}} \\
\Phi=\frac{\text { Vsolido }^{*} 100}{\text { Vpulpa }}
\end{gathered}
$$

$\mathrm{d}_{50}=$ Punto de corte en micrones $(\mu)$

Dc = Diámetro del ciclón (pulgadas)

$\mathrm{Di}=$ Diámetro alimentación (pulgadas)

Do = Diámetro overflow (pulgadas)

Du = Diámetro underflow

$\mathrm{Q}=$ Caudal $\left(\mathrm{ft}^{3} / \mathrm{min}\right)$

$\Phi=$ Porcentaje volumétrico del sólido

$h=$ Distancia entre la parte inferior del vortex y la superior del ápex (pulgadas).

$\rho_{s}=$ Gravedad específica del mineral

$\rho_{f}=$ Gravedad específica del líquido

Para realizar una selección de tamaños de una pulpa que contiene partículas de -200 mallas de la serie Tyler, el fluido es introducido bajo presión por la abertura del alimentador, la entrada tangencial hace que la pulpa gire dentro del ciclón, el diseño del aparato hace que una porción de líquido junto con las partículas de rápido asentamiento sean descargadas por la abertura del apex, el resto del líquido junto con las partículas de asentamiento lento son descargados por la salida del vortex.

\subsection{Restricciones en la aplicación}

1. La geometría del ciclón está fijada para dar un límite de separación de partícula en un rango útil.

2. El ciclón es arreglado para dar un reciclado continuo del producto del apex.

3. La relación entre el tamaño límite y el tamaño efectivo de separación de las partículas puede ser definida exactamente en términos de números de ciclos o al tiempo de funcionamiento bajo condiciones constantes.

4. El agua es usada como el medio líquido. Ver Tabla 1. 
TABLA 1

\begin{tabular}{|c|c|c|}
\hline \multicolumn{3}{|c|}{$\begin{array}{l}\text { CORRECCIÓN DE LA VISCOSIDAD POR } \\
\text { TEMPERATURA }\end{array}$} \\
\hline $\begin{array}{c}\text { Temperatura } \\
{ }^{\circ} \mathrm{C}\end{array}$ & $\begin{array}{l}\text { Viscosidad } \\
\text { Centipoises }\end{array}$ & $\begin{array}{l}\text { Factor de } \\
\text { Corrección }\end{array}$ \\
\hline 5 & 1.5188 & 1.2290 \\
\hline 10 & 1.3077 & 1.1410 \\
\hline 11 & 1.2713 & 1.1250 \\
\hline 12 & 1.2363 & 1.1090 \\
\hline 13 & 1.2028 & 1.0940 \\
\hline 14 & 1.1709 & 1.0790 \\
\hline 15 & 1.1404 & 1.0650 \\
\hline 16 & 1.1111 & 1.0510 \\
\hline 17 & 1.0826 & 1.0380 \\
\hline 18 & 1.0559 & 1.0250 \\
\hline 19 & 1.0299 & 1.0120 \\
\hline 20 & 1.0050 & 1.0000 \\
\hline 21 & 0.9810 & 0.9880 \\
\hline 22 & 0.9579 & 0.9760 \\
\hline 23 & 0.9358 & 0.9650 \\
\hline 24 & 0.9141 & 0.9540 \\
\hline 25 & 0.8937 & 0.9430 \\
\hline 26 & 0.8737 & 0.9320 \\
\hline 27 & 0.8545 & 0.9220 \\
\hline 28 & 0.8360 & 0.9120 \\
\hline 29 & 0.8180 & 0.9020 \\
\hline 30 & 0.8007 & 0.8930 \\
\hline 40 & 0.6560 & 0.8080 \\
\hline 50 & 0.5494 & 0.7390 \\
\hline
\end{tabular}

Fuente: Sepor INC "Ciclosizer For Sub-Sieve Sizing"

\section{MÉTODO}

3.1 Análisis de partículas ultrafinas mineral aurífero y dolomita usando el Cyclosizer

El Cyclosizer Warman Internacional es un equipo diseñado y construido para realizar el análisis granulométrico de partículas ultrafinas, menores a 44 micrones y los parámetros de operación son:

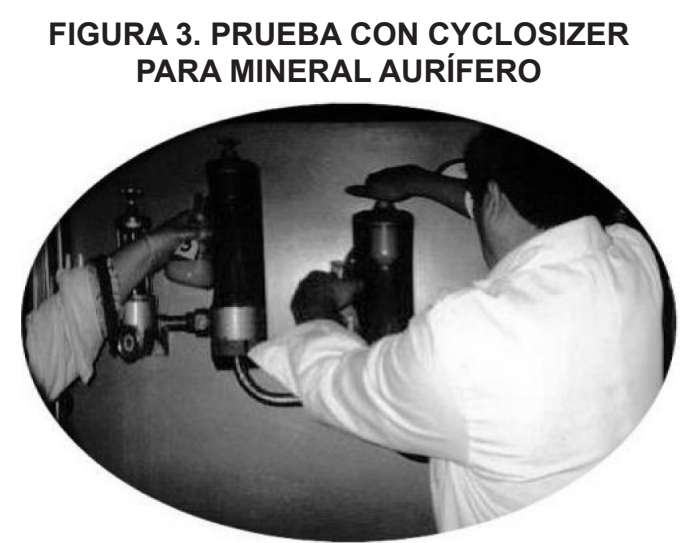

Silvana Flores Chávez Alfonso Romero Baylón David Martínez Aguilar

- Caudal de flujo

- Temperatura del agua

- Densidad de la partícula

- Tiempo de elutriación

TABLA 2

\begin{tabular}{|c|c|c|}
\hline \multicolumn{3}{|c|}{$\begin{array}{c}\text { RANGO DE SEPARACIÓN EN } \\
\text { CONDICIONES STANDARD }\end{array}$} \\
\hline CICLÓN & MICRONES & ERROR \\
\hline 1 & 44 & \pm 2 \\
2 & 33 & \pm 1 \\
3 & 23 & \pm 1 \\
4 & 15 & \pm 1 \\
5 & 11 & \pm 1 \\
\hline
\end{tabular}

FUENTE: Elaboración propia.

\section{Factores de ajuste}

$d e=d i * f_{1} * f_{2} * f_{3} * f_{4}$

de = Tamaño efectivo de separación de partículas.

Di = Tamaño límite de separación de partículas.

$\mathrm{F}_{1}=$ Factor de corrección de la temperatura del agua del ensayo y su lectura.

$\mathrm{F}_{2}=$ Factor de corrección de la gravedad específica de la partícula y su lectura.

$\mathrm{F}_{3}=$ Factor de corrección de la razón de flujo y su lectura.

$\mathrm{F}_{4}=$ Factor de corrección del tiempo de elutriación y su lectura.

\subsection{Prueba con el Cyclosizer Mineral aurífero}

TABLA 3

\begin{tabular}{|l|l|}
\hline \multicolumn{2}{|c|}{ CONDICIONES DE OPERACIÓN } \\
\hline Muestra & Mineral aurífero \\
\hline Peso & 30 gramos \\
\hline Temperatura & $20^{\circ} \mathrm{C}$ \\
\hline Tiempo de elutriación & $6 \mathrm{~min} .55 \mathrm{seg}$. \\
\hline Gravedad específica & $2.85 \mathrm{gr} / \mathrm{cc}$ \\
\hline Caudal de agua & $13.8 \mathrm{lt} / \mathrm{min}$ \\
\hline
\end{tabular}

TABLA 4

FACTOR DE CORRECCIÓN (FC)

\begin{tabular}{|c|l|l|}
\hline$f_{1}$ & Temperatura & 0.955 \\
\hline$f_{2}$ & Gravedad específica & 0.910 \\
\hline$f_{3}$ & Caudal de agua & 0.918 \\
\hline$f_{4}$ & Tiempo de elutriación & 0.940 \\
\hline
\end{tabular}

TABLA 5

\begin{tabular}{|c|c|c|}
\hline $\begin{array}{c}\text { CICLÓN } \\
\mathbf{N}^{\circ}\end{array}$ & $\begin{array}{c}\text { TAMAÑO DE } \\
\text { PARTÍCULA }\end{array}$ & $\begin{array}{c}\text { PESO } \\
\text { (gramos) }\end{array}$ \\
\hline 1 & $44^{*} 0.745=33 \mu$ & 2.00 \\
\hline 2 & $33^{*} 0.745=25 \mu$ & 3.00 \\
\hline 3 & $23^{*} 0.745=17 \mu$ & 5.70 \\
\hline 4 & $15^{*} 0.745=11 \mu$ & 4.80 \\
\hline 5 & $11^{*} 0.745=8 \mu$ & 4.70 \\
\hline
\end{tabular}


FIGURA 4

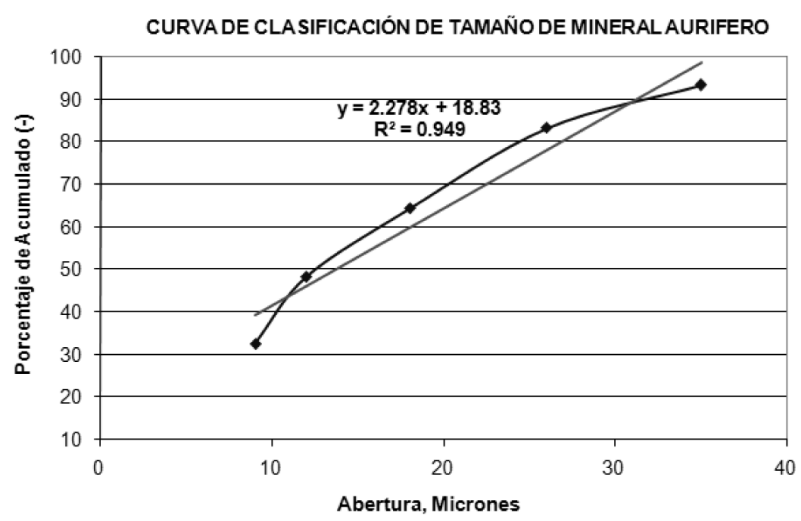

Figura 5. Prueba con cyclosizer para Mineral de Dolomita.

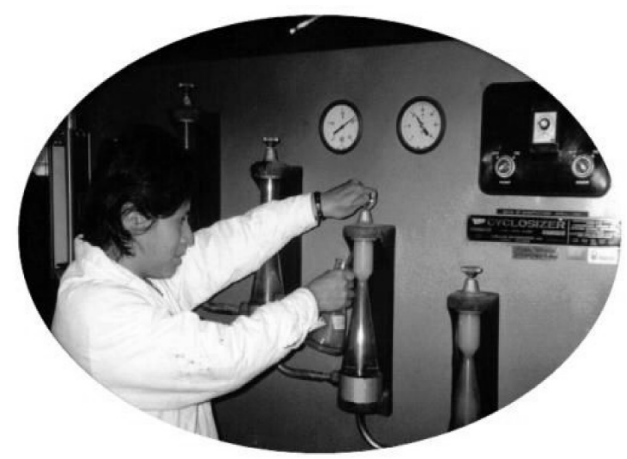

\subsection{PRUEBA CON EL CYCLOSIZER}

\section{MINERAL DOLOMITA}

TABLA 6

\begin{tabular}{|l|l|}
\hline \multicolumn{2}{|c|}{ CONDICIONES DE OPERACIÓN } \\
\hline Muestra & Mineral Dolomita \\
\hline Peso & 30 gramos \\
\hline Temperatura & $20^{\circ} \mathrm{C}$ \\
\hline Tiempo de elutriación & $2 \mathrm{~min} .30 \mathrm{seg}$. \\
\hline Gravedad específica & $2.15 \mathrm{gr} / \mathrm{cC}$ \\
\hline Caudal de agua & $13.8 \mathrm{lt} / \mathrm{min}$ \\
\hline
\end{tabular}

TABLA 7

\section{FACTOR DE CORRECCIÓN (FC)}

\begin{tabular}{|l|l|l|}
\hline \multicolumn{3}{|l|}{ FACTOR DE CORRECCIÓN (FC) } \\
\hline $\mathrm{f}_{1}$ & Temperatura & 0.955 \\
\hline $\mathrm{f}_{2}$ & Gravedad específica & 0.910 \\
\hline $\mathrm{f}_{3}$ & Caudal agua & 0.918 \\
\hline $\mathrm{f}_{4}$ & Tiempo de elutriación & 0.940 \\
\hline
\end{tabular}

$F C=* f_{1}^{*} f_{2}^{*} f_{3}^{*} f_{4}$

$F c=0.955 * 0.910 * 0.918 * 0.940$

$F_{c}=0.745$

TABLA 8

\begin{tabular}{|c|c|c|}
\hline $\begin{array}{c}\text { CICLÓN } \\
\mathbf{N}^{\circ}\end{array}$ & $\begin{array}{c}\text { TAMAÑO DE } \\
\text { PARTíCULA }\end{array}$ & $\begin{array}{c}\text { PESO } \\
\text { (gramos) }\end{array}$ \\
\hline 1 & $44^{*} 0.745=33 \mu$ & 2.00 \\
\hline 2 & $33^{\star} 0.745=25 \mu$ & 3.00 \\
\hline 3 & $23^{\star} 0.745=17 \mu$ & 5.70 \\
\hline 4 & $15^{\star} 0.745=11 \mu$ & 4.80 \\
\hline 5 & $11^{\star} 0.745=8 \mu$ & 4.70 \\
\hline
\end{tabular}

\section{FIGURA 6}

CURVA DE CLASIFICACIÓN DE TAMAÑO DE PARTICULA DE MINERAL DE DOLOMITA

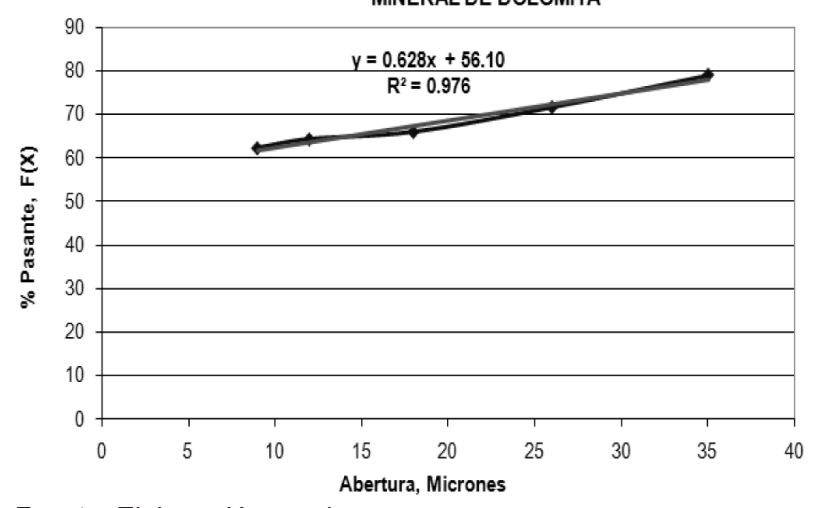

Fuente: Elaboración propia.

\section{DISCUSIÓN DE RESULTADOS}

\subsection{Resultado de prueba con el Cyclosizer Mineral aurífero}

TABLA 9

\begin{tabular}{|c|c|c|c|c|c|}
\hline CICLON & $\begin{array}{c}\text { TAMAÑO } \\
(\boldsymbol{\mu m})\end{array}$ & $\begin{array}{c}\text { PESO } \\
(\mathbf{g r})\end{array}$ & $\begin{array}{c}\% \\
\text { PESO }\end{array}$ & $\mathbf{G}(\mathbf{x})$ & $\mathbf{F}(\mathbf{x})$ \\
\hline $\mathrm{N}^{\circ} 1$ & 33 & 2.00 & 6.67 & 6.67 & 93.33 \\
\hline $\mathrm{N}^{\circ} 2$ & 25 & 3.00 & 10.00 & 16.67 & 83.33 \\
\hline $\mathrm{N}^{\circ} 3$ & 17 & 5.70 & 19.00 & 35.67 & 64.33 \\
\hline $\mathrm{N}^{\circ} 4$ & 11 & 4.80 & 16.00 & 51.67 & 48.33 \\
\hline $\mathrm{N}^{\circ} 5$ & 8 & 4.70 & 15.67 & 67.33 & 32.67 \\
\hline$-\mathrm{N}^{\circ} 5$ & & 9.80 & 32.67 & 100.0 & 0.00 \\
\hline & & 30.00 & 100.0 & & \\
\hline
\end{tabular}

4.2. Resultado de prueba con el cyclosizer de dolomita

TABLA 10

\begin{tabular}{|l|l|l|l|l|l|}
\hline CICLON & $\begin{array}{l}\text { TAMAÑO } \\
(\boldsymbol{\mu m})\end{array}$ & $\begin{array}{l}\text { PESO } \\
(\mathbf{g r})\end{array}$ & \% PESO & $\mathbf{G}(\mathbf{x})$ & $\mathbf{F}(\mathbf{x})$ \\
\hline $\mathrm{N}^{\circ} 1$ & 33 & 6.30 & 21.00 & 21.00 & 79.00 \\
\hline $\mathrm{N}^{\circ} 2$ & 25 & 2.20 & 7.33 & 28.33 & 71.67 \\
\hline $\mathrm{N}^{\circ} 3$ & 17 & 1.70 & 5.67 & 34.00 & 66.00 \\
\hline $\mathrm{N}^{\circ} 4$ & 11 & 0.50 & 1.67 & 35.67 & 64.33 \\
\hline $\mathrm{N}^{\circ} 5$ & 8 & 0.60 & 2.00 & 37.67 & 62.33 \\
\hline$-\mathrm{N}^{\circ} 5$ & & 18.70 & 62.33 & 100.00 & 0.00 \\
\hline & & 30.00 & 100.00 & & \\
\hline
\end{tabular}

Fuente: Elaboración propia. 
El factor de corrección del tamaño de clasificación del cyclosizer es de 0.745 para el mineral aurífero y para la dolomita.

El $32.67 \%$ del mineral aurífero y el $62.33 \%$ del mineral de dolomita se encuentra a un tamaño menor a $8 \mu \mathrm{m}$.

El coeficiente de correlación de la distribución granulométrica del mineral aurífero es de 0.9494 y el de la dolomita de 0.9768 .

\section{CONCLUSIONES}

Las partículas finas son efectivamente elutriadas, en las dos muestras ensayadas, en función de un flujo ascendente de aire.
Silvana Flores Chávez Alfonso Romero Baylón David Martínez Aguilar

La elutriación es función del tamaño y tipo de respuesta de la partícula a la fluidización neumática, de modo tal que aumenta con la velocidad superficial del aire.

El arrastre se hace mayor para las muestras que tienen una mayor cantidad de finos presentes.

\section{REFERENCIAS BIBLIOGRÁFICAS}

1. Sepor INC

"Cyclosizer For Sub-Sieve Sizing".

2. Jaime M. Tapia

"Separación Neumática de Finos desde Corrientes de Mineral Chancado".

3. Iván Quiroz Núñez, "Ingeniería Metalúrgica”. 